\title{
QUALIDADE MICROBIOLÓGICA DE LEITE DE TANQUE DE REBANHOS LEITEIROS DO ESTADO DE ALAGOAS, BRASIL
}

ALVES, Elizabeth Simões do Amaral ${ }^{1}$

SANTOS, Micheline Thais dos ${ }^{2}$ PEIXOTO, Aila Fabiane ${ }^{3}$ NUNES, Kleber Barros ${ }^{3}$

SILVA, João Manoel da ${ }^{4}$

SILVA, Paula Cibelly Vilela da ${ }^{5}$

SANTOS, Tania Marta Carvalho dos ${ }^{6}$

MEDEIROS, Elizabeth Sampaio de ${ }^{7}$

Recebido em: 2020.04.24 Aprovado em: 2021.03.08 $\quad$ ISSUE DOI: $10.3738 / 21751463.3787$

\begin{abstract}
RESUMO: O objetivo desse estudo foi avaliar a qualidade microbiológica de amostras de leite cru resfriado proveniente de três regiões do estado de Alagoas (Litoral, Agreste e Sertão). De cada fazenda foi coletado 2 tipos de amostras de leite: amostras dos quartos mamários e amostras do leite de tanque. Os rebanhos eram constituídos de animais de diferentes raças, idades e estágios de lactação. Foram realizados ensaios para contagem de microorganismos mesófilos, psicrotróficos, coliformes totais e termotolerantes e Staphylococcus spp.. As amostras de leite resfriado apresentaram os micro-organismos em estudo, evidenciando que para esse tipo de leite, as amostras estão estão às margens dos padrões estabelecidos pela legislação vigente quanto à qualidade microbiológica. As amostras de leite resfriado apresentaram mesófilos $\left(>3 \log _{1} 0_{0} \quad \mathrm{UFC} / \mathrm{ml}\right)$, psicrotróficos $\left(<1 \log _{1} 0_{0} \mathrm{UFC} / \mathrm{ml}\right)$ e coliformes totais $\left(3,6\right.$ a $>1100 \log _{1}$ o NMP/ml) e termotolerantes $\left(3\right.$ a $>1100 \log _{1}$ o UFC/ml), além da alta frequência de Staphylococcus spp. (2,8 a >3 $\left.\log _{1} 0 \mathrm{UFC} / \mathrm{ml}\right)$. A presença desses micro-organismos pode estar associada às más práticas de manejo de ordenha, bem como higiene do ambiente e deficiências na refrigeração, o que pode ocasionar deterioração do produto e contaminação dos animais e consumidores finais.
\end{abstract}

Palavras-chave: Staphylococcus. Coliformes. Mesófilos. Psicrotróficos. Qualidade alimentar.

\section{MICROBIOLOGICAL QUALITY OF TANK MILK FROM DAIRY HERDS OF ALAGOAS STATE, BRAZIL}

SUMMARY: The aim of this study was to evaluate the microbiological quality of chilled raw milk samples from three regions of the state of Alagoas (Litoral, Agreste and Sertão). From each farm, 2 types of milk samples were collected: samples from the mammary quarters, adn samples of tank milk. Herds were made up of animals of different breeds, ages and stages of lactation. Tests were carried out to count mesophilic, psychrotrophic microorganisms, total and thermotolerant coliforms and Staphylococcus spp.. The samples of chilled milk presented the microorganisms under study, showing that for this type of milk, the samples are on the margins of the standards established by the current legislation regarding microbiological quality. The chilled milk samples showed mesophiles (>3 $\left.\log _{1} 0 \mathrm{CFU} / \mathrm{ml}\right)$, psychrotrophic $\left(<1 \log _{1} 0 \mathrm{CFU} / \mathrm{ml}\right)$ and total $\left(3.6\right.$ to $\left.>1100 \log _{1} 0 \mathrm{MPN} / \mathrm{ml}\right)$ and thermotolerant coliforms ( 3 to $>1100 \log _{1}$ o $\quad \mathrm{UFC} / \mathrm{ml}$ ), in addition to the high frequency of Staphylococcus spp. (2.8

\footnotetext{
${ }^{1}$ http://orcid.org/0000-0001-5078-4104

Programa de pós-graduação em Produção Animal, Centro de Ciências Agrárias, Universidade Federal de Alagoas.

2 http://orcid.org/0000-0002-7675-2554

Programa de pós-graduação em Biociência Animal, Universidade Federal Rural de Pernambuco.

${ }^{3}$ Programa de pós-graduação em Produção Animal, Centro de Ciências Agrárias, Universidade Federal de Alagoas.

${ }^{4}$ http://orcid.org/0000-0002-7654-5475 Engenheiro Agrônomo, Mestre em Ciências. Experiência em Microbiologia Agrícola e Ambiental e Microbiologia de Alimentos. Rede Nordeste de Biotecnologia, Instituto de Química e Biotecnologia, Universidade Federal de Alagoas

http://orcid.org/0000-0003-2157-4698

Programa de pós-graduação em Produção Animal, Centro de Ciências Agrárias, Universidade Federal de Alagoas.

${ }^{6}$ http://orcid.org/0000-0002-1816-7840 Campus de Engenharias e Ciências Agrárias, Centro de Ciências Agrárias, Universidade Federal de Alagoas

${ }^{7}$ http://orcid.org/0000-0002-1289-2902 Departamento de Medicina Veterinária, Universidade Federal Rural de Pernambuco.
} 
to $>3 \log _{1}$ o $\left.\mathrm{UFC} / \mathrm{ml}\right)$. The presence of these microorganisms may be associated with poor milking management practices, as well as environmental hygiene and deficiencies in refrigeration, which can cause deterioration of the product and contamination of animals and final consumers.

Keywords: Staphyococcus. Coliforms. Mesophiles. Psycrotrophic. Food health.

\section{INTRODUÇÃO}

O leite é um alimento produzido e consumido mundialmente. No Brasil, o leite é um dos principais produtos agropecuários, contribuindo para o desenvolvimento econômico dos locais em que a atividade é praticada. No ano de 2018, o país produziu 2.069 litros/vaca/ano de leite, com crescimento de $10 \%$ no Nordeste. O estado de Alagoas está inserido na principal microrregião produtora de leite, responsável por $25 \%$ do total produzido na região (ZOCCAL, 2019).

A cadeia de produção de laticínios vincula as propriedades de produção de leite com associações e centros de processamento e beneficiamento, aumentando o valor do produto em cada etapa da cadeia produtiva até chegar no consumidor final. O início da cadeia de laticínios normalmente envolve a produção, o armazenamento e transporte do leite até os centros de processamento (FAO, 2016).

No Brasil, a atividade leiteira apresenta diferentes sistemas de produção de acordo com a região em que o leite é produzido e a condição de renda dos produtores, tais singularidades resultam na utilização de diferentes tipos manejo, raça dos animais, especialização ou não na ordenha e armazenamento do leite na fazenda, volume produzido, entre outros (SOUTO et al., 2009). Ademais, embora existam especificações técnicas para produção de leite, nem sempre estas são seguidas em sua totalidade. Em Alagoas, a região Agreste importância para o setor lácteo alagoano, possuindo influência no aumento da produtividade leiteira do estado (SANTANA; SILVA; VIEIRA, 2016)

$\mathrm{O}$ armazenamento do leite produzido nas pequenas e médias propriedades é feito em tanques de resfriamento para posterior coleta e transporte a granel. Apesar de reduzir perdas econômicas por atividade acidificante de bactérias mesófilas, essa prática permite a seleção de bactérias psicrotróficas relacionadas a problemas tecnológicos e econômicos na indústria de laticínios (FONSECA; SANTOS, 2000). Assim, para a legislação vigente, permite-se que o leite possa ser armazenado sob resfriamento entre 4 a $7{ }^{\circ} \mathrm{C}$ por período máximo de $48 \mathrm{~h}$ (BRASIL, 2018).

Nucleus Animalium, v.13, n.1, maio 2021 
A composição microbiológica do leite é reflexo de práticas higiênico-sanitárias adotadas em todos os setores produtivos da cadeia leiteira. A legislação brasileira vigente, estabelece parâmetros microbiológicos de identidade e requisitos mínimos de qualidade para os alimentos industrializados, como o leite pasteurizado e o leite Ultra High Temperature (UHT), contudo micro-organismos podem contaminar o leite desde a saída deste do úbere, na manipulação pelos funcionários, pelos equipamentos e utensílios utilizados, durante o transporte, durante o processamento, dentre outros (MAPA, 2011; MATA; TOLEDO; PAIVA, 2012; SALVADOR et al., 2012; TEIXEIRA; FIGUEIREDO, 2019).

Diante do exposto, objetivou-se por meio desse estudo avaliar a qualidade microbiológica do leite individual e do leite do rebanho leiteiro produzido em fazendas de três regiões do estado de Alagoas.

\section{MATERIAL E MÉTODOS}

\section{Descrição da área experimental}

O presente estudo foi realizado em 3 rebanhos de propriedades rurais sendo cada uma localizada nas respectivas mesorregiões do Agreste, Sertão e Litoral de Alagoas, Brasil, que de acordo com a classificação de Köppen (1948) possuem: Litoral, clima Ams', tropical com chuvas de outono a inverno e médias pluviométricas anuais entre $1.500 \mathrm{~mm}$ a $2.200 \mathrm{~mm}$ e temperatura média do ar variando entre $23^{\circ} \mathrm{C}$ e $28^{\circ} \mathrm{C}$; Agreste, apresenta condições semiáridas, com clima BSh, isto é, seco e quente, com precipitação pluviométrica média anual no Agreste de $600 \mathrm{~mm}$ a $900 \mathrm{~mm}$ com temperatura média do ar variando entre $21^{\circ} \mathrm{C}$ a $23^{\circ} \mathrm{C}$ e no Sertão entre $400 \mathrm{~mm}$ a $600 \mathrm{~mm}$ com temperatura média do ar variando entre $17^{\circ} \mathrm{C}$ e $33^{\circ} \mathrm{C}$.

De cada fazenda foi coletado 2 tipos de amostras de leite: amostras dos quartos mamários e amostras do Leite de tanque de resfriamento. Os rebanhos eram constituídos de animais de várias raças, idades e diferentes estágios de lactação, sendo adotados 20 animais em cada propriedade, resultando em amostras compostas.

\section{Amostragem}

Para a coleta de leite individual, as amostras de leite dos quartos mamários foram colhidas após prévia lavagem do teto com água e sabão, secagem com papel toalha e antissepsia do óstio do teto com álcool a $70{ }^{\circ} \mathrm{GL}$. Em continuidade obteve-se um volume de $10 \mathrm{ml}$, em frascos com tampa rosqueável, esterilizados e previamente identificados com o nome ou número do animal bem como o quarto mamário correspondente. Estas amostras foram acondicionadas à temperatura de refrigeração em caixa isotérmica, contendo gelo reciclável e encaminhadas ao laboratório para as devidas análises. 
Uma alíquota de $0,1 \mathrm{ml}$ foi semeada em ágar base acrescido de $10 \%$ de sangue de ovino desfibrinado. As placas foram incubadas a $37^{\circ} \mathrm{C}$, em estufa microbiológica, realizando-se as leituras após 24 horas. Os agentes isolados foram identificados de acordo com as características morfológicas de suas colônias, como tamanho, tipo, coloração e presença de hemólise. Ao microscópio, foram observadas a disposição das células e as características morfotintorias à técnica de Gram (SPECK, 1976; SIQUEIRA, 1995).

Logo após ordenha de todo o rebanho leiteiro, foi realizado a coleta do leite do tanque diretamente no tanque de expansão refrigerado $\left(4{ }^{\circ} \mathrm{C}\right.$ com temperatura previamente dimensionada) após $2 \mathrm{~h}$ de armazenamento, em frascos estéreis, os quais foram transportados até o laboratório em caixas térmicas com gelo reciclável, permanecendo sob refrigeração $\left(4{ }^{\circ} \mathrm{C}\right)$ durante o transporte. As amostras corresponderam a amostras compostas por região.

No laboratório, as amostras de leite foram pesadas e diluídas da seguinte forma: $25 \mathrm{ml}$ da amostra adicionadas a $225 \mathrm{ml}$ de solução salina peptonada $0,1 \%$, diluições até $10^{-3}$ para contagem de Staphylococcus spp., mesófilos e psicrotrófilos e $10^{-1}$ para a pesquisa de Coliformes Totais e Termotolerantes (SPECK, 1976; SIQUEIRA, 1995).

Para a contagem de $S$. aureus, as amostras diluídas foram inoculadas na superfície de placas contendo ágar Baird-Parker. Após o término das operações, as placas foram incubadas à temperatura de $37^{\circ} \mathrm{C}$, por 48 horas. Foram contadas as placas que continham entre 20 e 200 colônias suspeitas de S. aureus. Os resultados foram expressos em unidades formadoras de colônias (UFC) de S. aureus por ml da amostra.

Em seguida, foi realizado ensaio para detecção de mesófilos e psicrotrófilos, onde as placas foram incubadas conforme os micro-organismos, sendo a temperatura de $(32+2)^{\circ} \mathrm{C}$ para mesófilos; $(7 \pm 2)^{\circ} \mathrm{C}$ para psicrotrófilos por 24 horas, sendo obrigatória a inoculação em superfície. O resultado foi expresso de acordo com o número de colônias, em unidade formadora de colônias (UFC).

Para a pesquisa de Coliformes Totais e Fecais, as amostras foram inoculadas em tubos contendo caldo Lauril Sulfato Triptose (LST), considerando resultado positivo os que apresentaram gás nos tubos de Duhram. A confirmação da presença de Coliformes Totais foi feita por meio da inoculação das colônias que cresceram em caldo Verde Brilhante Bile Lactose VB) $2 \%$ e posterior incubação a $36 \pm 2{ }^{\circ} \mathrm{C}$ por 24 a 48 horas. A presença de gás nos tubos de Durhan do caldo VB evidenciou a fermentação de lactose presente no meio. A confirmação da presença de Coliformes Termotolerantes foi feita por meio da incubação em caldo EC, com incubação em temperatura $45 \pm 2{ }^{\circ} \mathrm{C}$ em banho-maria com agitação por 24 . Foram considerados positivos os Nucleus Animalium, v.13, n.1, maio 2021 
tubos que apresentaram gás nos tubos de Durhan. Os resultados foram expressos por presença ou ausência de coliformes totais e termotolerantes. Todas as análises supracitadas foram desenvolvidas de acordo com o Manual de Análise de Alimentos (SPECK, 1976).

\section{Análises estatísticas}

O delineamento experimental foi inteiramente casualizado. Os dados coletados foram submetidos à análise de variância (ANAVA) p $\leq 0,05$ pelo teste $F$ no software Sisvar 5.6 (Ferreira, 2014).

\section{RESULTADO E DISCUSSÃO}

Foi observada altas contagens de bactérias aeróbias mesófilas foram nas amostras de leite cru refrigerado, o que pode ser reflexo de falhas nas práticas higiênico-sanitárias adotadas na ordenha, nos utensílios, no armazenamento ou na refrigeração durante a estocagem (SEQUETTO et al., 2017).

Nesse estudo, a contagem de organismos psicotróficos foi menor que 1,0x $10^{3} \mathrm{UFC} / \mathrm{ml}$ nas amostras de leite cru refrigerado (Tabela 1). Apesar de não haver legislação específica para a contagem de bactérias psicotróficas no Brasil, segundo Beloti (2015) a contagem de psicrotrófilos deve ser de, no máximo, $10 \%$ da contagem total de aeróbios mesófilos.

Tabela 1 - Contagem de micro-organismos mesófilos e psicrotóficos em leite cru refrigerado de rebanhos leiteiros do estado de Alagoas, Brasil.

\begin{tabular}{cccc}
\hline \multirow{2}{*}{ Região } & \multirow{2}{*}{ Amostras } & \multicolumn{2}{c}{ Micro-organismos* } \\
\cline { 3 - 4 } & & $\begin{array}{c}\text { Mesófilos } \\
\left(\log _{1} 0 \quad \mathrm{UFC} / \mathrm{ml}^{3}\right)\end{array}$ & $\begin{array}{c}\text { Psicrotróficos } \\
\left(\log _{1}{ }_{0}\right.\end{array}$ \\
\hline Litoral & $\mathrm{LCR}^{1}$ & $>3,00$ & $<1,0$ \\
\hline Agreste & LCR & $>3,00$ & $<1,0$ \\
\hline Sertão & LCR & $>3,00$ & $<1,0$
\end{tabular}

${ }^{1}$ LCR: Leite cru refrigerado; ${ }^{2}$ Unidades Formadoras de Colônia por mililitro. *Valores seguidos de mesma letra não diferem estatisticamente entre si pelo teste de Tukey $(\mathrm{p} \leq 0,05)$.

Fonte: Elaboração dos autores.

A presença de bactérias psicrotróficas no leite cru está associada aos procedimentos inadequados de higienização do maquinário durante a produção, tempo e temperatura em que o leite é armazenado. A baixa contagem de psicrotróficos no leite é um importante apontador de qualidade, devido à atividade metabólica desses micro-organismos que resulta em alterações bioquímicas nos constituintes do leite que diminuem a vida útil do leite fluido e seus derivados (MARIOTO et al., 2020). 
Micro-organismos mesófilos foram detectados apenas nas amostras de leite cru resfriado, com valores acima de $3 \log _{10} \mathrm{UFC} / \mathrm{ml}^{-1}$, o que demonstra uma deficiência sanitária nesse tipo de leite, embora a Legislação vigente (IN 37 2000). Ademais, esse grupo de micro-organismos possui a habilidade de crescimento em temperatura ambiente e fazem parte da microbiota natural do leite (GUEDES-CELESTINO et al., 2019), porém, cargas altas da população podem ocasionar em deterioração do leite. Outro fator importante é que, embora pertençam à microbiota natural, seu crescimento em ambiente refrigerado é ocasionado por inadequações na refrigeração e o pré armazenamento.

No presente estudo foi verificada uma contagem de coliformes totais e termotolerantes acima de $1100 \mathrm{NMP} / \mathrm{ml}$ para as amostras coletadas no Litoral. As menores contagens foram observadas nas amostras oriundas do Sertão (Tabela 2). Os coliformes quando encontrados em amostras de leite são indicadores da presença de resíduos de fezes (BRITO; BRITO; PORTUGAL, 2002), o que pode ser ocasionado ainda durante a ordenha, como também a higiene total do ambiente onde é realizada.

Tabela 2 - Contagem de coliformes totais e termotolerantes e Staphylocossus spp. em leite cru refrigerado de rebanhos leiteiros do Litoral, Sertão e Agreste alagoanos.

\begin{tabular}{lcccc}
\hline Região & Amostras & $\begin{array}{c}\text { Coliformes totais } \\
\left(\mathbf{N M P} / \mathbf{m l}^{\mathbf{3}}\right.\end{array}$ & $\begin{array}{c}\text { Coliformes } \\
\text { termotolerantes } \\
(\mathbf{U F C} / \mathbf{m L})^{\mathbf{4}}\end{array}$ & $\begin{array}{c}\text { Staphylococcus spp. } \\
(\mathbf{U F C} / \mathbf{m l})\end{array}$ \\
\hline Litoral & LCR $^{\mathbf{1}}$ & $>1100$ & $>1100$ & $>3,00 \times 10^{3}$ \\
\hline Agreste & LCR & 150 & $<3,0$ & $1,21 \times 10^{3}$ \\
\hline Sertão & LCR & 3,6 & $<3,0$ & $2,8 \times 10^{3}$ \\
\hline
\end{tabular}

1'LCR: Leite cru refrigerado; ${ }^{2}$ UHT: Ultra Hight Temperature; ${ }^{3}$ UFC: Unidades Formadoras de Colônia por mililitro; ${ }^{4}$ Número Mais Provável por mililitro.

Fonte: Elaboração dos autores.

Staphylococcus spp. foi detectado com contagens a partir de 1,21 x $10 \mathrm{UFC} / \mathrm{ml}^{3}$ (Tabela 2), sendo observado apenas nas amostras de leite cru refrigerado, onde as amostras UHT não apresentaram crescimento de nenhum micro-organismo, o que evidencia a eficiência do processo. A supracitada bactéria é um patógeno associado ao complexo que causa mastite em animais como bovinos e caprinos (BARROS et al., 2018), podendo também estar associado à deterioração do leite e causar doenças transmitidas por alimentos, conhecidas como DTA's.

Ademais, outros micro-organismos também estão associados à mastite e DTA's. Os resultados do presente estudo constataram a presença de Streptococcus, spp., Corynebacterium 
spp., Micrococcus spp., e cocos Gram - (Tabelas 3 e 4), os quais são descritos na literatura como causadores de DTA's e associados à mastite (BARROS et al., 2018; TEIXEIRA; FIGUEIREDO, 2019). Passos et al. (2012) afirmam que aproximadamente $41 \%$ dos surtos de origem alimentar estão relacionados ao $S$. aureus. A importância clínica da presença dessa bactéria é principalmente nos grupos de risco, sendo considerada como uma bactéria oportunista (TEIXEIRA; FIGUEIREDO, 2019).

Tabela 3 - Frequência absoluta e relativa das bactérias isoladas dos quartos mamários dos rebanhos leiteiros do Litoral, Agreste e Sertão alagoanos.

\begin{tabular}{cccc}
\hline Região & Bactérias Isoladas & $\mathrm{FA}^{1}(\%)$ & $\mathrm{FR}^{2}(\%)$ \\
\hline & Staphylococcus spp. & 11 & 42,3 \\
& Streptococcus spp. & 10 & 38,46 \\
Litoral & Corynebacterium spp. & 1 & 3,84 \\
& Micrococcus spp. & 2 & 7,69 \\
& Cocos Gram - & 2 & 7,69 \\
\hline \multirow{5}{*}{ Agreste } & Staphylococcus spp. & 10 & 62,5 \\
& Streptococcus spp. & 4 & 25 \\
& Micrococcus spp. & 2 & 12,5 \\
\hline \multirow{5}{*}{ Sertão } & Staphylococcus spp. & 7 & 50 \\
& Streptococcus spp. & 5 & 35,71 \\
& Micrococcus spp. & 1 & 7,14 \\
& Cocos Gram - & 1 & 7,14 \\
\hline
\end{tabular}

1FA: Frequência absoluta; ${ }^{2}$ FR: Frequência relativa.

Fonte: Elaboração dos autores.

Staphylococcus spp. foi a bactéria com maior frequência (Tabela 4) encontrada nas amostras analisadas no presente estudo, sendo encontrada apenas nas amostras de leite cru resfriado. Esse é um dos patógenos mais comumente encontrados em amostras de leite cru. Ressalta-se que a presença desse micro-organismo pode levar a intoxicações alimentares e se torna uma preocupação para a saúde pública (FAGUNDES et al., 2010).

Tabela 4 - Bactérias isoladas nos rebanhos leiteiros no Litoral, Agreste e Sertão alagoanos.

\begin{tabular}{ccc}
\hline $\mathbf{X}^{\mathbf{1}}$ & $\mathbf{n}(\mathbf{x})^{\mathbf{2}}$ & $\mathbf{P}(\mathbf{x})^{\mathbf{3}}$ \\
\hline Staphylococcus spp. & 28 & 50 \\
Streptococcus spp. & 19 & 33,92 \\
Corynebacterium spp. & 1 & 1,78 \\
Micrococcus spp. & 5 & 8,92 \\
Cocos Gram - & 3 & 5,35 \\
Total & 56 & 99,97 \\
\hline
\end{tabular}

${ }^{1 X}$ : Bactéria; ${ }^{2} \mathrm{n}(\mathrm{x})$ : Frequência absoluta; ${ }^{3 \mathrm{P}(\mathrm{x})}$ : Frequência relativa.

Fonte: Elaboração dos autores. 
O controle de qualidade dos alimentos, desde a obtenção da matéria prima até a elaboração do produto final como queijos e outros alimentos, envolve ações e medidas de controle, tais como treinamento de manipuladores de acordo com o regimento do Manual de Boas Práticas, monitoramento e fiscalização dos mesmos, bem como dos processos de acondicionamento dos alimentos e higienização desses (FERREIRA; DIAS; JÁCOME JUNIOR, 2016).

Maior parte dos micro-organismos isolados são de origem exógenas ao animal, sendo, portante, fundamental atentar-se ao manejo da ordenha e sanitização do ambiente onde vive o animal, bem como dos manipuladores. Além da sanidade do animal, preza-se também pela sanidade dos consumidores, uma vez que esses micro-organismos, em parte alguns oportunistas, que possuem a capacidade de causar DTA's em humanos.

Quando encontradas em concentrações acima de $10^{6} \mathrm{UFC} / \mathrm{ml}$ essas bactérias podem produzir enzimas termorresistentes, capazes de gerar proteólise e lipólise nos nutrientes que compõem o leite, o que ocasiona a redução da vida útil do produto e possível dano à saúde do consumidor (SAEKI; MATSUMOTO, 2010).

\section{CONCLUSÃO}

As regiões produtoras de leite apresentam diferenças em sua qualidade microbiológica quando se comparadas as amostras de leite cru resfriado, onde apresentam contagem altas de micro-organismos, embora estes não estejam listados na legislação vigente

Staphylococcus spp. é a bactéria mais abundante e frequente nas amostras de leite estudadas.

As amostras de leite UHT apresentaram características microbiológicas excelentes, onde todas mostraram ausência de micro-organismos.

\section{REFERÊNCIAS}

BARROS, A. F.; ALVES, E. S. A.; SILVA, J. M.; DOS SANTOS, T. M. C. Diagnóstico e etiologia de mastite subclínica em caprinos leiteiros. Ciência Agrícola, v. 16, suplemento, p 1-3, 2018. Doi: 10.28998/rca.v16i0.6602

BELOTI, V. Leite: Obtenção, Inspeção e Qualidade. Londrina: Planta, 2015, 480 p. 
BRASIL. Ministério da Agricultura, Pecuária e Abastecimento. Instrução Normativa n. 76.

Diário Oficial da União. Brasília: MAPA, 2018. Available on: <http://www.in.gov.br/materia/lasset_publisher/Kujrw0TZC2Mb/content/id/52750137/do1-2018-11-30-instrucao-normativa-n76-de-26-de-novembro-de-2018-52749894IN\%2076>. Accessed march, 20, 2020.

BRASIL. Ministério de Agricultura, Pecuária e Abastecimento - MAPA. Instrução Normativa n. 62, de 29 de dezembro de 2011. Diário Oficial da União. Brasília, D. Seção 1, 30 de dez de 2011.

FAGUNDES, H.; BARCHESI, L.; NADER FILHO, A.; FERREIRA, L. M.; OLIVEIRA, C. A. F. Ocorrência de Staphylococcus aureus em leite cru produzido em fazendas leiteiras no estado de São Paulo, Brasil. Brazilian Journal of Microbiology, v. 41, n. 2, 2010. Doi: 10.1590/S0103-84782004000400058

FERREIRA, D. F. Sisvar: a Guide for its Bootstrap procedures in multiple comparisons. Ciência e Agrotecnologia, v. 38, n. 2, p. 109-112, 2014. Doi. http://dx.doi.org/10.1590/S1413-70542014000200001

FERREIRA, A. L.; DIAS, M. B. N.; JÁCOME JÚNIOR, A. T. Avaliação da contaminação por Staphylococcus aureus, Coliformes e Salmonella sp. em espetinhos comercializados por ambulantes no centro de Caruaru-PE. 2016, 12f. Trabalho de Conclusão de Curso (Graduação em Biomedicina) Faculdade Asces, Caruaru, PE, 2016.

FONSECA, L. F. L.; SANTOS, M. V. Qualidade do leite e controle de mastite. São Paulo: Lemos, 2000.

Food And Agriculture Organization Of The United Nations - FAO. Organizational challenges and the institutional environment: a comparative analysis of dairy cooperatives in Kenya and Morocco, 2016. Disponível em <http://www.fao.org/publications/card/en/c/f98ed155-d025-479cb6d7-5825a0ae7d7f >. Acesso em: 22 abr. 2020.

GUEDES-CELESTINO, E.; SANTOS, M. T.; SILVA, S. G. M.; SANTOS, T. M. S.; ALVES, E. S. M.; MELO, P. A.; MELO, B. A. Microbiological quality of goat milk produced on Alagoas state, Brazil. Nucleus Animalium, v. 11, n. 2, p. 47-55, 2019. Doi: 10.3738/21751463.3613

KÖPPEN, W. Climatologia: com un estudio de los climas de la tierra. Mexico: Fondo de Cultura Economica, 1948.

MARIOTO, L. R. M.; DANIEL, G. C.; GONZAGA, M; MAREZE, J.; TAMANINI, R.; BELOTI, V. Potencial deteriorante da microbiota mesófila, psicrotrófica, termodúrica e esporulada do leite cru. Ciência Animal Brasileira, v.21, p. e-4434, 2020. Doi: 10.1590/1809$6891 v 21 \mathrm{e}-44034$

MATA, N. F.; TOLEDO, P. S.; PAVIA, P. C. A importância da pasteurização: comparação microbiológica entre leite cru e pasteurizado, do tipo B. Revista do Instituto Laticínios Cândido Tostes, v. 67, n. 384, p. 66-70, 2012. Doi: 10.5935/2238-6416.20120010

PASSOS, E. C.; ALMEIDA, A. S.; MELLO, A. R. P. Presença de Bacillus cereus, Clostridium perfringens, Staphylococcus aureus e Escherichia coli em surto de toxinfecção alimentar ocorrido na região do Vale do Ribeira. Revista do Instituto Adolfo Lutz, v. 71, n. 4, p. 713-717, 2012.

SAEKI, E. K.; MATSUMOTO, L. S. Contagem de mesófilos e psicrotróficos em amostras de leite pasteurizado e UHT. Revista do Instituto de Laticínios Cândido Tostes, v. 65, n. 377, p. 29-35, 2010. 
SALVADOR, F. C.; BURIN, A. S.; FRIAS, A. A. T.; FAILA, N. Avaliação da qualidade microbiológica do leite pasteurizado e comercializado em Apucarana - PR e região. Revista F@pciência, v. 9, n. 5, p. 30-41, 2012.

SEQUETTO, P. L.; ANTUNES, A. dos S.; NUNES, A. S.; ALCANTARA, L. K. S.; REZENDE, M. de R., PINTO, M. A. de O.; FONTES, G. G.; HÚNGARO, H. M. Avaliação da qualidade microbiológica de leite cru refrigerado obtido de propriedades rurais da zona da mata mineira. Revista Brasileira de Agropecuária Sustentável, v. 7, n. 1, p. 42-50, 2017. Doi: 10.21206/rbas.v7i1.388

SOUTO, L. I. M.; SAKATA, S. T.; MINAGAWA, C. Y.; TELLES, E. O.; GARBUGLIO, M. A.; BENITE, N. R. Qualidade higiênico-sanitária do leite cru produzido em propriedades do estado de São Paulo, Brasil. Arquivo Brasileiro de Medicina Veterinária e Zootecnia, v. 16, n. 3, p. 491-499, 2009.

SANTANA, R. S.; SILVA, J. C. S.; VIEIRA, F. A. Produção de leite em Microrregiões do Agreste Alagoano. NutriTime, v. 13, n. 6, p. 4891-4894, 2016.

SIQUEIRA, R. S. Manual de Microbiologia de Alimentos. Centro Nacional de Pesquisa de Tecnologia Agroindustrial de Alimentos. Rio de Janeiro: EMBRAPA - CTAA, 1995, 159p.

SPECK, M. L. Compendium for the microbiological examination of foods. Washington, D. C: American public health association, 1976, 701p.

TEIXEIRA, M. S.; FIGUEIREDO, M. A. Qualidade microbiológica do leite bovino no Brasil associada à Staphylococcus aureus. Revista de Ciência Veterinária e Saúde Pública, v. 6, n. 1, p. 196-216, 2019. Doi: 10.4025/revcivet.v6i1.41172

ZOCCAL, R. Leite nas grandes regiões brasileiras. In: Anuário Leite, Embrapa Gado de Leite. p. 50-58. 2019. Disponível em <https://www.embrapa.br/gado-de-leite>. Acesso em 21 abr. 2020. 